\title{
Good results of chronic HCV management in a single centre prison population
}

\author{
Authors: Hisham Sherfi, Sheila Needs, Keith George and James Neale
}

\section{Introduction}

It is a rapidly evolving era for the management of hepatitis $\mathrm{C}$ with very high cure rates. There is an increasing need to identify, diagnose and treat this difficult and vulnerable group of patients. It is paramount that these patients have an access to hepatology services and treatment. $32 \%$ of already diagnosed patients in our region were prisoners.

\section{Aims}

To assess our experience and outcome in managing patients with chronic hepatitis C. Also to compare this outcome between prisoners and the general population with the same disease.

\section{Methods}

We retrospectively collected data on already-treated HCV patients between 2005 and 2012 in our centre. All the patients were treated with PEG-IFN $\alpha$ plus ribavirin. We looked into genotype, treatment naïve or experienced, cirrhotic or not, side effects and compliance. As in 2005 and 2006 patients were either classified into genotypel or non-1, we continued with the same classification for the whole study.

\section{Results}

The total number of patients was 217, out of these 53 were prisoners (32\%).

18 of the prisoners were genotype1, out of which 13 were cured $(72 \%)$. The rest of them (35) were non-1 and 29 were cured $(83 \%)$. Three out of the 53 prisoners were cirrhotic, all genotype non-1 and all cured. All patients in this group were treatment naïve. Two were lost to follow-up due to release and one stopped treatment due to side effects.

Regarding the other group (non-prisoners), 68 were genotypel and 33 were cured (49\%). The remaining (96) were non-1 and 71 were cured (74\%). 20 patients of this group were cirrhotic (10 genotypel and 10 non-1 and four were cured from each group). 10 patients were lost to follow-up, five stopped due to side effects and one continued to inject heroin. Only one patient was treatment experienced, cirrhotic and genotype1 and was cured.

The imprisoned patients have had a noticeable better cure rate, especially in genotype 1 (chi-square test with continuity correction; $\mathrm{p}=0.07$ ) and less so for non-1 group.

\section{Conclusions}

Obviously in our centre we are having a reasonably good access to approach and treat prisoners with chronic hepatitis $\mathrm{C}$. This group tends to comply well with treatment regime hence better outcome. We need further improvement to follow up/hand over patients moved to different prisons to assure continuity of care. 